Sādhanā, Vol. 19, Part 1, February 1994, pp. 51-73. (C) Printed in India.

\title{
A fuzzy expert system approach using multiple experts for dynamic follow-up of endemic diseases
}

\author{
APURBA BANERJEE, ARUN KUMAR MAJUMDER ${ }^{1}$ and \\ ANUPAM BASU ${ }^{2}$ \\ Department of Computer Science \& Engineering, Indian Institute of \\ Technology, Kharagpur 721 302, India
}

E-mail: 1anupam@cse.iikgp.ernet.in; 2akmj@cse.iitkgp.ernet.in

\begin{abstract}
In this paper, an architecture of LEPDIAG - a knowledge-based system for on-line diagnosis and for monitoring prognosis of leprosy is presented. The important features of LEPDIAG that have been detailed are a multiple expert environment, a homeostatic expert containing the model of immune reaction, a performance evaluator that can compare the observed signs and symptoms with those predicted by the homeostatic expert and a prognostic expert which optimizes the management schedule for the patients. The entire systems is built around a fuzzy expert-system building tool FEXT to deal with the imprecise knowledge.
\end{abstract}

Keywords. Homeostasis; idiotypic network; immunological reaction.

\section{Introduction}

The advent of expert systems has concretised the advantage of artificial intelligence, in the form of practical systems. Expert systems have been applied to a number of generic task domains such as diagnosis (Chilausky et al 1976; Davis et al 1977; Chandrasekharan et al 1979), planning (Martin 1977), design (McDermott \& Steele 1981), mineral exploration (Hart et al 1978) etc. A good review of expert system organisation and its applicability in different domains is available in Stefic et al (1982). The boom of expert system activities in medical diagnosis was set off by the seminal work on MYCIN (Buchanan \& Shortliffe 1984) and was followed by a number of important developments like INTERNIST, ONCOCIN, TEIRESIAS etc. (Buchanan \& Shortliffe 1984). Medical diagnosis activities also led to interesting architectures (Clancey 1983; Davis 1983; Reggia \& Tuhrim 1985; Patil 1987; Sticklen 1987).

Our investigations in this area convinced us that the domain of medical diagnosis demands the use of multiple expert modules instead of a monolithic structure of the first generation expert systems. The task of medical diagnosis treads into a number of decision activities of varying nature, ranging from abductive diagnosis, tentative

A glossary of medical terms used is provided at the end of the paper 
prognosis as well as a deep analyses of the particular status of the homeostatic equilibrium. Hence, for proper diagnosis and also for a reasonable attempt to model the cognitive process of a medical expert, it is essential to have an environment, where each of these areas of expertise is captured and allowed to work in close coordination.

Another aspect closely associated with medical diagnosis in the uncertainty of knowledge and data. Different approaches for dealing with uncertainty in the medical domain has been proposed such as CF in MYCIN (Buchanan \& Shortliffe 1984), belief function (Shafer 1976) etc. The imprecise correlation between signs and symptoms with disease can be elegantly captured by fuzzy reasoning technique (Zadeh 1983a). A number of medical expert systems have adopted fuzzy techniques (Albin 1975; Perez-Ojeda 1976; Adlassnig \& Kolarz 1982, pp. 219-47; Cios et al 1991).

In this paper, we present the architecture and reasoning techniques of LEPDIAG - an expert system for dynamic follow-up of endemic diseases.

The problem that is often encountered in chronic endemic diseases, such as leprosy, tuberculosis, AIDS etc., is the changes in the symptom and sign complex of a disease over time. Due to inherent immunological reactions in the body, it is expected that the symptom pattern will undergo considerable changes with change in the state of a disease. The effect of such changes become more pronounced as a consequence of medication given in the early stages of the disease. The development of a diagnostic system which addresses this problem requires an adequate model of the dynamics of the symptom-disease evolution pattern. In this paper, we have represented the immunological information and its effect on symptom-disease relationship in the form of rules. This approach enables us to deal with the changes in symptom-disease relationship over time and to interact with the conventional rule-based approach to medical diagnosis. In our current discourse, we intend to deal with a typical endemic disorder - leprosy.

LEPDIAG provides a diagnostic environment, involving multiple expert modules working in close coordination, through a shared framebase. The knowledge base consists of fuzzy rules clustered in functional partitions. The inference machine supports fuzzy inferencing and is implemented through a fuzzy expert system building tool FEXT.

For a proper understanding of the problem domain, a brief introduction to the disease is presented in the next section.

\section{Leprosy as a disease}

The most remarkable feature of leprosy is the wide variation of its manifestation depending on the immunological status of the individual. In same patients it may be manifested with a single nerve involvement or a single skin patch, while in others it may produce diffuse involvement of skin with multiple nodules together with polyneuritis and damage to the vital organs such as eyes, larynx, testes and bones. Every conceivable variation occurs between these two extremes. The causes of these variations lie in the variation of the patient's immune status and not merely on the bacterial strains with varying pathogenicity. In fact, it has been confirmed by Rees (1969) that leprosy bacilli from patients with different types of leprosy all behave in the same manner when injected into susceptible mice.

There are five clinical variations, seven histopathological and twelve immunohistological variations of the disease-complex (Ridley 1972). Each variation can be 
denoted as a distinct disease-state within the whole range of the leprosy diseasespectrum. A single patient can pass through all the five clinical variations before getting cured, or while suffering from mutilating miseries on a finite time scale. Moreover, time-framed changes of clinical, pathological and immunohistological parameter values provide enough information to detect resistant infection, inadequate drug therapy, or immunological instability leading to various types of lepra reactions.

\subsection{Evolution of signs and symptoms over time}

Essentially, a set of signs and symptoms of a specific disease is true only at that time instance and depends only on the current status of the homeostatic stability of the patient's body (Ganong 1989, pp. 34-5). Homeostatic stability depends upon the quantum and quality of the environmental injury, i.e. on antigens entering into the system, type and quantity of drugs administered, inappropriate metabolism of proximate principles, stress and strains modulating genetic information flow. It also depends on the concerted attempt of the multiple subsystems, namely immune system, vascular system, endocrine system etc., to contain the behaviour within the viable range of dynamic equilibrium.

With reference to leprosy, duration of the treatment varies from a minimum of five years to the entire lifetime of the patient. According to the immunological status of the individual, a leprosy patient may swing in the spectrum of different disease-states corresponding to polar tubercular leprosy at one end to polar lepromatous leprosy at the other extreme (Nath 1983). The states of a disease and the changes in symptom pattern over time are primarily dependent on the immune reactions in the body. Hence, to capture the dynamic relationships between symptoms and diseases, we need to have a suitable model of the immune system. In the following subsection an attempt has been made to develop such a pragmatic model which is useful for our purpose.

\subsection{Immune reaction model}

Although several models of immunodynamics have been presented over the years (Richter 1978, pp. 219-27; Herzenberg \& Black 1980), none of them is a useful tool to help in the diagnosis and prognosis of infective disorders. An interconnected idiotypic network model (Jerne 1973) can be accepted as a more feasible representative of the above class where the network provides control over the idiotypic interactions among the $T$-suppressors, $T$-helpers and the antibodies to any antigenic challenge, in any given immune system (Rich 1988). Details about the models can be found elsewhere (Richter 1978, pp. 219-27; Herzenberg \& Black 1980; Perelson 1989). In the present work, the behaviour of the idiotypic network is represented in terms of rules. The rule-based approach has been adopted so that the immune reaction model can be easily integrated with the diagnostic expert system. Moreover, a rule-based approach, especially with fuzzy rules, can readily accommodate the imprecise measurement and estimation of antibody concentration and T-lymphocyte population.

For the proposed work, two hundred and fifty patients of leprosy in the School of Tropical Medicine, Calcutta have been examined. It is seen from the available data that though the in-vivo lepromin test results appear to be a weak parameter in determining a patients's clinical status, other in-vitro tests such as the LTT (lymphocyte 
transformation test) etc. are potent enough in that respect. These observations indicate that the integral relationship among the antigenic load (BI), $T$-lymphocyte populations, index of T-lymphocyte suppression (LTT), and the level of antibodies is very irregular and complex. Available data also indicate that the manifestation of immunological parameters are time-dependent. Therefore, to represent the changes of the above parameters occurring over time and their corroboration with the manifested signs and symptoms, a rule-based model is necessary. An example rule of such a model is given below:

$R l$.

If

Antigen entering into the system is $A$

AND T-S population is very low

AND $T-H$ population is very high

Then

The quantity of the antibody $A b_{x}$ would be very low after a few days.

Such rules form the knowledge-base of the homeostatic expert HEXPERT, which has been entwined with a diagnostic expert system, a prognostic expert and a performance evaluator to form the composite system LEPDIAG. Thus it is possible to view the proposed system as a cooperating system of different knowledge sources, each dedicated to its own functional subtask. In the following section, we dwell on the detailed architecture of LEPDIAG and also describe the functions of each of the component expert systems. The interactions among these individual expert modules to form the composite whole of LEPDIAG is also explained.

\section{Overview of the proposed system (LEPDIAG)}

A schematic of LEPDIAG is shown in figure 1. The system consists of three expert system modules: diagnostic expert (DIAG), homoeostatic expert (HEXPERT), and prognostic expert (PROG) together with a performance evaluator (PERF).

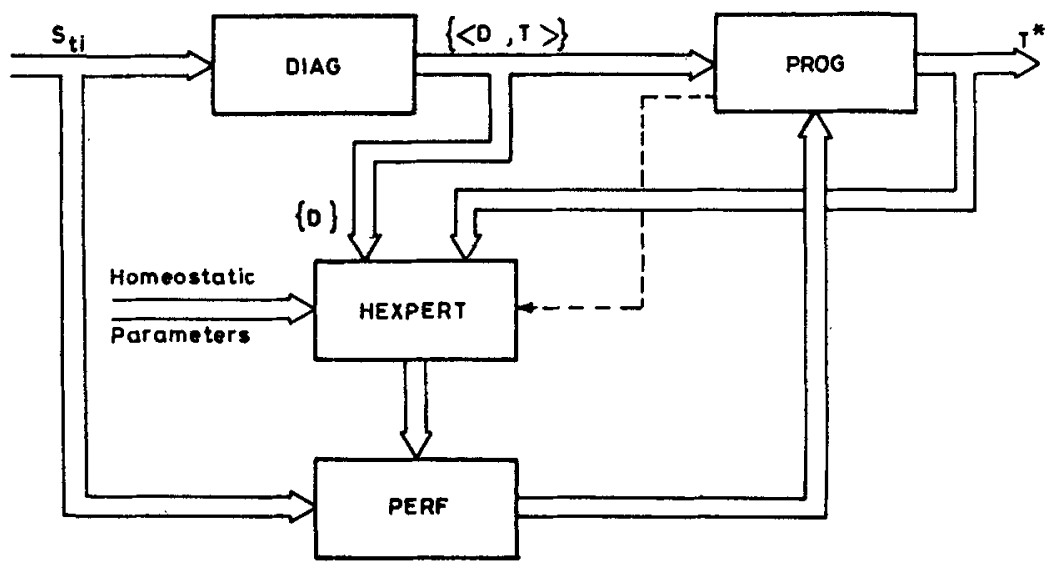

Figure 1. An overview of LEPDIAG. 
DIAG: It is a diagnostic and therapeutic expert, which accepts the signs and symptoms of the patient, at any point of time, as the input. Suppose that signs and symptoms identified at time $t$ are denoted by $S_{t}$. Based on the signs, and symptoms $S_{t_{0}}$ at time $t_{0}$, DIAG arrives at a list of possible diagnoses on utilizing the knowledgebase contained in it. Each identified disease is associated with a degree of belief and a list of candidate treatments. Thus the output of DIAG can be denoted by $\langle D, T\rangle_{t_{0}}$ where $D$ is a possible disease along with the associated belief and $T$ is a list of treatments for this disease. Each treatment is also associated with a confidence factor.

PROG: The prognostic expert accepts as input the set of diseases and treatments $\langle D, T\rangle_{t}$, identified by DIAG at time $t$ and utilises its prognostic knowledge-base to decide on the most likely disease and selects the most suitable treatment for it. The output $T_{t_{0}}^{*}$ of PROG is fed to HEXPERT for further refinement of the prognosis as discussed below.

HEXPERT: The knowledge-base of the homoeostatic expert contains the knowledge about the "dynamic behaviour" ( $\$ 2.1)$ of the respective disease. Given a set of possible diseases; administered treatments and the values of different homoeostatic parameters (such as lymphocyte count, bacterial index etc.) of the disease model, HEXPERT can predict some new signs and symptoms, which will be manifested after a predefined interval of time.

PERF: The performance evaluator module accepts inputs from two sources. The predicted set of signs and symptoms $\hat{S}_{t}\left(D, T^{*}, P_{h}, t_{0}\right)$, arrived at by HEXPERT is propagated to PERF. Also, as the patient visits the clinic at the next time instant (that is at time $\left.t_{1}\right)$, the actual set of signs and symptoms $S_{t_{1}}$, is observed. This set $\left(S_{t_{1}}\right)$ forms the second input of PERF. The performance evaluator compares $\hat{S}_{t_{1}}\left(D, T^{*}, P_{h}, t_{0}\right)$ and $S_{t_{1}}$ and finds the extent of match.

In the next section the organisation of the knowledge bases of the different modules are discussed.

\section{Knowledge modules}

The physical architechture of LEPDIAG is shown in figure 2. Different logical experts as shown in figure 1 are essentially a collection of knowledge modules partitioned in blocks of rule bases. Each block of knowledge module is dedicated to a particular phase of the entire task.

\subsection{DIAG}

The first expert, the diagnostic and therapeutic expert (DIAG) is a constellation of seven knowledge modules. In the first phase, the module $K B 1$ is utilised to identify a set of possible diseases from a set of patient data, termed as complaint. Each of the diagnoses arrived at is associated with a degree of belief with a fuzzy truth value to indicate the relative possibility of different diseases. It is often necessary to carry out checks to find out some other components of complaints which might have been omitted by the patient. To this end, DIAG utilises $K B 2$ to suggest further checks 


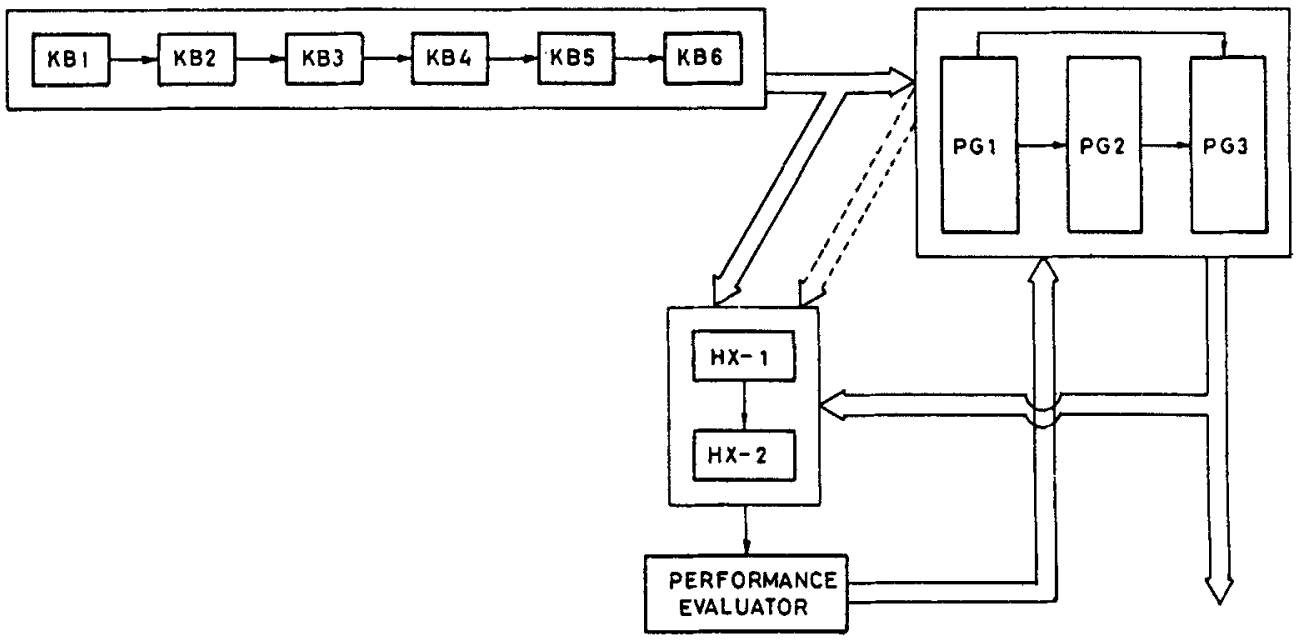

Figure 2. Physical architecture of LEPDIAG.

needed for the particular session. The check results thus obtained by the patient are utilised by the knowledge module $K B 3$ which generates a new set of facts with the modified belief of provisional diagnoses. The module $K B 4$ contains rules for suggesting special investigations like pathological and biochemical tests. There is a submodule $K B 4^{\prime}$ within $K B 4$ which examines from the list of beliefs about the diagnoses whether any such special investigations would be necessary for the session or not. The test results obtained through $K B 4$ are utilised by the knowledge module $K B 5$. This module basically analyses the investigation reports and modifies the associated belief of the provisional diagnoses accordingly, thus helping DIAG to arrive at a more confirmed set of provisional diagnoses. The task of diagnosis is basically abductive in nature. In. LEPDIAG the reasoning is essentially forward chaining. However, the abductive nature of reasoning has been easily captured by forward chaining rules associated with belief measures. In abduction $p \rightarrow q$ yields that if $q$ is true then $p$ is possibly true. In LEPDIAG such situations have been captured as $q$ (symptom) $\rightarrow p$ (disease rate) 〈fuzzy belief $\rangle$. For the current session, $K B 5$ also checks whether the belief about leprosy is significant enough and invokes $K B 6$ if leprosy has been diagnosed, otherwise the expert system halts, printing the diagnosis having maximum belief. Knowledge module $K B 6$ enquires about some more clinical facts in terms of check-signs to classify the patient to the respective slot of disease-state from which the patient is suffering at that time of diagnosis. Since leprosy involves a spectrum of diseases, $K B 6$ determines belief about the variations of leprosy at the current-time instant. Thus KB6 output consists of a set of leprosy-related diseases and their respective beliefs.

\subsection{HEXPERT}

The homoeostatic expert consists of two knowledge modules. The first module $H X 1$ gets the set of admissible diseases, $D$ from DIAG and a single treatment regime $T^{*}$ from the third expert PROG (discussed in \$4.4). The knowledge-base $H X 1$ is utilised to identify the investigations to be done for the internal homoeostatic parameters $\left(P_{h}\right)$ (viz., immunohistological parameters in any infective disorder such as leprosy) 
for each element of the set $D$. The second module $H X 2$ uses the values of the homoeostatic parameters $\left(P_{h}\right)$ along with the treatment $T^{*}$ for simulating the homoeostatic process. This module also enquires about the time interval for which the simulation should be done for the respective Ds. The module $H X 2$ calls the respective disease models for generation of predicted signs and symptoms with appropriate beliefs i.e., $\hat{S}_{t}\left(D, T^{*}, P_{h}, t_{0}\right)$ after the specified time interval.

\subsection{PERF - The evaluator}

The set of predicted signs and symptoms released by HEXPERT is taken up by the evaluator (PERF) - a submodule for checking (comparison of the match between the observed signs at time instant $t\left(S_{t}\right)$ and the predicted ones i.e., $\hat{S}_{t}\left(D, T^{*}, P_{h}, t_{0}\right)$. Comparison may lead to following two cases.

Case I: Suppose that the predicted and the observed set of signs and symptoms i.e., $\hat{S}_{t}\left(D, T^{*}, P_{h}, t_{0}\right)$ and $S_{t}$ match. While computing the degree of match, fuzzy beliefs about the predicted and observed symptoms are taken into account. The evaluator compares a predicted symptom $s$ which belongs to $\hat{S}_{t}\left(D, T^{*}, P_{h}, t_{0}\right)$ with a similar element $s$ belonging to $S_{r}$. If the each $S \in \hat{S}_{t}\left(D, T^{*}, P_{h}, t_{0}\right)$, there exists an $s \in S_{t}$ such that the similarity measure (cf. $\$ 5.3$ ) about their belief is above the threshold, the PERF signals the "match" to PROG. But if the value of the similarity measure is less than the predefined threshold value then evaluator conveys the "mismatch" to PROG to initiate appropriate steps.

For example, in a case of borderline leprosy as a possible diagnosis, suppose that the initial signs and symptoms at time $t_{0}$ (at time of first examination) are as follows:

(1) number of skin patches: considerable;

(2) shine over the skin lesions: moderate;

(3) bilateral symmetry of the lesions: less marked;

(4) area of loss of sensation: large.

Patients's information about immunohistological parameters are as follows:

(1) bacterial index less than 3;

(2) lepromin test $1+$;

(3) lymphocyte transformation test more than $3 \%$;

(4) antibody titre moderately high.

After treatment with Dapsone and Rifampicin for one month, the expected set of signs and symptoms $\left(\hat{S}_{\text {onemoneh }}\right)$ are:

(1) number of skin patches: small number;

(2) shine over the skin lesions: absent;

(3) bilateral symmetry: absent;

(4) area of loss of sensation: moderately large.

When the patient returns after one month, the observed set of signs and symptoms are:

(1) number of patches: moderately high;

(2) shine over the lesions: less moderate;

(3) bilateral symmetry: absent;

(4) area of loss of sensation: large. 
Table 1. Initial signs and symptoms and their fuzzy attributes.

\begin{tabular}{lc}
\hline Initial signs and symptoms & Fuzzy attributes \\
\hline 1. Right ulnar nerve thickening & Marked \\
2. Single patch over right thumb & Large \\
3. Loss of sensation & Severe \\
\hline
\end{tabular}

Interestingly, no new symptoms or signs have been palpable after one month. The evaluator (PERF) compares the beliefs of the respective signs and symptoms in the predicted and observed sets.

Case II: There may be some occasions when for some $S \in \hat{S}_{t}\left(D, T^{*}, P_{k}, t_{0}\right)$ there would be no corresponding $s$ belonging to $S_{\mathrm{r}}$. Similarly, for some $s \in S_{\text {, no }}$ no corresponding $s$ belonging to $\hat{S}_{1}\left(D, T^{*}, P_{n}, t_{0}\right)$ may be present. These two cases are dealt as follows:

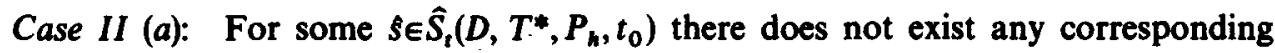
$s \in S_{t}$. Example: In a case of type I lepra reaction at time $t_{0}$ the legs and face of the patient were moderately swollen (oedema: moderate), there was severe aching all over the joints and there was neurological deficit grade II. On treatment with Clofazimine $(300 \mathrm{mg}$ ) daily, Ibuprofen (400) twice daily, vitamin B-complex and adequate antacid coverage for seven days, the expected set of signs and symptoms $\hat{S}_{7}$ becomes:

Oedema: less marked; aching: less acute; and neurological deficit: very low.

Suppose that after seven days the following observations $\left(S_{7}\right)$ are reported:

Oedema: moderate; and aching: moderate.

Note that the observed set does not include any information about the third symptom. Therefore, there is difficulty in computing a straightforward "match" or "mismatch" by the evaluator. This problem is resolved by the evaluator which recommends information regarding the missing symptom to be gathered. Once necessary information is fed, the matching process is repeated again.

Case II (b): For some $s \in S_{t}$; there does not exist any corresponding $\hat{S}_{t} \hat{S}_{t}\left(D, T^{*}, P_{h}, t_{0}\right)$. For example, in a case of borderline tubercular leprosy the observations made are reported in table 1.

On treatment with Dapsone and Rifampicin for one month, the expected set $\hat{S}_{\text {onemonth }}$ would be as given in table 2 .

Table 2. Predicted signs and symptoms and their fuzzy attributes.

\begin{tabular}{ll}
\hline Predicted signs and symptoms & Fuzzy attributes \\
\hline 1. Right ulnar nerve thickening & Less marked \\
2. Patch over right thumb & Moderate \\
3. Loss of sensation & Moderate \\
\hline
\end{tabular}


Table 3. Actual signs and symptoms and their fuzzy attributes after one month.

\begin{tabular}{ll}
\hline Actual signs and symptoms after one month & Fuzzy attributes \\
\hline 1. Right ulnar nerve thickening & More marked \\
2. Patch over right thumb & Large \\
3. Loss of sensation & Moderate \\
4. Tenderness over right ulnar nerve & Marked \\
5. Aching over the joints & Mild to moderate \\
\hline
\end{tabular}

On observation after seven days the actual set of signs and symptoms $S_{\text {onemomn }}$ contains elements as listed in table 3.

Here two new signs and symptoms (4) and (5) appear in the set $S_{\text {onemonth }}$ which were altogether absent in $\hat{S}_{\text {onemonth }}$. The evaluator (PERF) takes some extra care to deal with such cases. Firstly, it computes the similarity measure among the remaining elements of $\hat{S}_{t}\left(D, T^{*}, P_{k}, t_{0}\right)$ and $S_{t}$ and modifies the belief of previous provisional diagnosis in PROG by sending a "match" or "mismatch". Simultaneously, it takes the help of HEXPERT through the module PG1 in PROG (described below) to find another disease $d_{k} \in D_{t_{0}}$ for which the predicted and the observed set of symptoms match. The process is repeated till no disease exists in $D_{t_{0}}$ for which the predicted and observed symptoms are found to match.

\subsection{PROG}

The expert PROG has been divided into three knowledge modules. The first module $P G 1$ interacts with the evaluator module. It gets the input from the evaluator which identifies whether a "match" or "mismatch" has occurred between the predicted and the observed symptoms.

Suppose that the evaluator has sent a match between $\hat{S}_{t}\left(D, T^{*}, P_{h}, t_{0}\right)$ and $S_{t}$ to $P G 1$ which in turn activates $P G 2$. At the same time $P G 2$ also receives the admissible

Table 4. Frames and attributes used in LEPDIAG.

\begin{tabular}{ll}
\hline List of frames & \multicolumn{1}{c}{ Example attributes } \\
\hline Frame Control & Status \\
Complaints & Chronic Ulcer \\
Checksigns & Regional Anaesthesia \\
InvestI & Blood Sugar (PP) \\
InvestlI & Skin Smears \\
PRDI & Tabes Dorsalis \\
PRDII & Pytiriasis Versicolor \\
PRDIII & Lepra ReactionI \\
Immune Status & Bacterial Index \\
Leprosy & Borderline Lepromatous \\
General Status & Intercurrent Infection \\
Drug & Clofazimine \\
\hline
\end{tabular}


set of $\left\langle D, T^{*}\right\rangle_{t}$ from DIAG in the forward loop at the time instant $t$. Since the evaluator has indicated a match between $\hat{S}_{t}\left(D T^{*}, P_{h}, t_{0}\right)$ and $S_{t}, P G 2$ then increments the belief about the disease $D$ in the provisional diagnosis $D_{t}$ arrived at by DIAG. If $T^{*}$ is the treatment recommended by DIAG at time $t$ for the disease $D$ i.e., $\left\langle D T^{*}\right\rangle \in\langle D, T\rangle_{t}$, the belief about $T^{*}$ is also incremented. PG2 then examines the elements of newly generated $\langle D, T\rangle$, with their associated beliefs. Finally, the disease and treatment pair $\left(D^{*}, T^{*}\right)$ having highest belief is selected and passed to the next module PG3.

The evaluator sends a "mismatch" to PG1. The module communicates to the homoeostatic expert for finding the predicted set of symptoms $\hat{S}_{t}\left(D T^{*}, P_{h}, t_{0}\right)$ for an other $d_{k}$ in the set $D_{t_{0}}$. This process is repeated till all the elements of $D_{t_{0}}$ are exhausted or a single case of "match" is arrived at by the evaluator. If a "match" appears, it is dealt with as described above. But if all the elements of $D$ are exhausted without finding a "match", then the forward loop predominates and $P G 2$ only accepts $\left\langle D, T^{*}\right\rangle$ supplied by DIAG at time $t$.

The knowledge module $P G 3$ is concerned with the therapeutics of different diseases. The module $P G 3$ receives as input the diagnosed disease-entity and its treatment from PG2. This module also enquires about patient's general status and drug toxicity parameters. It then optimizes the drug elements (along with the adjustment of doses of different drugs for different individuals). For example, if the most possible diagnosis as given by the module $P G 2$ is borderline leprosy and the proposed drug no. 1 is Dapsone then PG3 asks for the following facts:

(1) whether any tenderness is present over the adjoining peripheral nerve;

(2) whether there is any past history of lepra reaction present in the patient;

(3) whether the skin patches are looking considerably inflamed;

(4) whether the patient is running a temperature;

(5) percentage of haemoglobin in the blood sample of the patient.

Suppose the replies to the queries for a particular patient are as follows:

(1) Considerable; (2) history of lepra reaction three years earlier; (3) slight; (4) mild; (5) $10 \mathrm{gm} \%$.

Then the module PG3 indicates that:

(1) administration of steroid $20 \mathrm{mg}$ daily is indicated;

(2) oral administration of Clofazimine $300 \mathrm{mg}$ daily to be started;

(3) oral antacids and antiflatulents are to be administered;

(4) to report to the expert after one week.

\section{Knowledge representation and inferencing}

The information entities in LEPDIAG are organized in the form of objects. Each such object consists of a number of attributes. These objects and attributes are implemented as frames and slots. A list of typical objects are enumerated in table 1.

LEPDIAG has been built around an expert system building tool FEXT. FEXT (fuzzy expert tool) is an enhanced version of DEXT (Basu et al 1988) and incorporates the features required for dealing with fuzzy information and knowledge. In FEXT expert directives and heuristics are encoded through production rules. These domain specific rules are stored in the rulebase in the form of 'condition-action' type of rules. The 
rules are represented as

$$
\text { Rule }::=\mathrm{IF}\langle\text { antecedent field }\rangle \mathrm{THEN}\langle\text { consequent field }\rangle\langle\mathrm{BM}\rangle,
$$

where $\langle B M\rangle$ stands for belief measure, which is a real number or a fuzzy qualifier indicating a heuristic weight or fuzzy truth value associated with a rule. The working memory of FEXT has been organized as a collection of "frames". FEXT views every feature of a problem as an information object which may consist of several component pieces of information.

Belief measure (BM): In certain situations, the state of the working memory (WM) may match the antecedent field of more than one rule, resulting in a conflict among the match rules. The conflicting rules often suggest different diagnoses, decision algorithms etc. In such cases, the expert's heuristics can be applied for resolving the conflict. The expert's heuristic preferences are encoded in the form of belief measures associated with the rules. BM represents the confidence or belief of the expert in the truth of a rule. As detailed in $\$ 5 \cdot 1, \mathrm{BM}$ relates to $\tau$, the truth qualifier of a rule. Note that $\tau$ can be a crisp value or a fuzzy set. Consequently, in FEXT, BM can be coded either as a positive real number or in terms of a fuzzy linguistic qualifier and the composite belief about the multiple facts obey the principle of arithmetic operations on fuzzy numbers (Kandel 1986; Leung \& Lam 1988). The implementation of BM is in sharp contrast to the confidence factor (CF) used in MYCIN and others where only real numbers have been used as CF and the composite belief has been computed as a simple multiplication of the CF. The methodology for computing such beliefs has been explained in $\S 5 \cdot 1$. This measure also is utilized for resolving conflicts in conjunction with meta rules as explained in $\$ 5 \cdot 2$.

FEXT allows the rules to be considered as a number of rule clusters depending on the functional characteristics of the rules. Also special constructs are available in FEXT for intercluster communication and context switching. Thus in the context LEPDIAG, FEXT facilitates the modelling of different knowledge modules as distinct rule clusters.

Example rules of LEPDIAG: This rule belongs to rule cluster II, rule no. 7.

Then

IF ( $\$$ checksigns \# skin patch = flat)

(\$checksigns \# patch pigment $=$ hypo)

Set (\$ PRDII \# vitiligo = considerable)

AND Set (\$PRDII \# pytiriasis-versicolor = likely)

AND Set (\$ PRDI \# leprosy = more likely).

The rule should be read as if the observed skin patch over the patient's body is flat and hypopigmented then the belief about the diagnoses of vitiligo, pytiriasisversicolor and leprosy would be 'considerable', 'likely' and 'more likely' respectively.

The inference machine of FEXT adopts forward chaining of inferencing. Also, in tune with the medical domain, the knowledge is often imprecise and fuzzy. The fuzzy inferencing required by LEPDIAG is captured by FEXT as discussed below.

\subsection{Fuzzy inferencing}

Definitions and concepts about the fuzzy set theory are available in the literature (Kaufman 1975). In the fuzzy set literature, a rule If $X$ is $A$ then $Y$ is $B$ is treated 
as a conditional fuzzy proposition which defines a fuzzy relation over $U \times V$. There are several alternative translation schemes for defining the fuzzy relation associated with a conditional fuzzy proposition (Zadeh 1983b).

In LEPDIAG, we have used the translation rule $R_{g}$ defined below to determine the possibility distribution associated with a conditional fuzzy proposition.

\section{DEFINITION}

Let $F$ and $G$ be fuzzy subsets of $U$ and $V$, respectively. The possibility distribution $\pi(X \rightarrow Y)$ associated with the conditional fuzzy proposition If $\mathbf{X}$ is $\mathbf{F}$ then $\mathbf{Y}$ is $\mathbf{G}$ is given by

$$
\pi(X \rightarrow Y)=R_{g}
$$

where $R_{g}$ is a fuzzy subset of $U \times V$ with membership function

$$
\mu_{R_{g}}(u, v)=1 \text {, if } \mu_{F}(u)<\mu_{G}(v)=\mu_{G}(v) \text {, otherwise. }
$$

Suppose that $A$ and $B$ are fuzzy sets over the universes $U$ and $V$ respectively. Consider a fuzzy rule: If $X$ is $A$ then $Y$ is $B$. Suppose that one intends to use this rule with a fact $X$ is $A 1$ where $A 1$ is a fuzzy set over $U$. By generalised modus ponens (Nilsson 1982), one can infer:

\section{Ant1: If $X$ is $A$ then $Y$ is $B$ \\ Ant2: $X$ is $A 1$ \\ Cons: $Y$ is $B 1$}

where $B 1$ is a fuzzy set over $V$. The possibility $B 1$ of $Y$ is computed using the translation rule $\boldsymbol{R}_{g}$ for conditional fuzzy proposition and the compositional rule of inference (Mitzumoto and Zimmerman 1982). Accordingly, the membership function for $B 1$ is given by:

$$
\mu_{B 1}(y)=\max _{x}\left(\min \left(\mu_{A 1}(x), \mu_{R_{q}}(x, y)\right)\right. \text {. }
$$

When the rule is truth qualified such as if $X$ is $A$ then $Y$ is $B$ is $\tau$, where $\tau$ is a fuzzy set over [0.1], then with a fact $X$ is $A 1$ the possibility distribution $B 1$ of the consequent is obtained by applying the translation rule for truth qualified propositions and compositional rule of inference. Thus we have:

If $X$ is $A$ then $Y$ is $B$ is $\tau$ Rule

$$
X \text { is } A 1 \text { Fact }
$$

\section{$Y$ is $B 1$ Consequent}

The membership function of $B 1$ is given by:

$\mu_{B 1}(y)=\left(\mu_{s}\right)_{x}\left(\max \left(\min \left(\mu_{A 1}(x), \mu_{R_{g}}(x, y)\right)\right)\right)$. We can generalize the inference procedure to the cases where a rule involves more than one antecedent. For instance, consider a rule:

If $X_{1}$ is $A_{1}$ and $X_{2}$ is $A 2$ and,.., $X_{k}$ is $A k$ then $Y$ is $B$.

In this case, the translation rule defines a fuzzy relation $R_{g}$ over $U_{1} \times U_{2}, \ldots$, $U_{k} \times V$ where $U_{i}$ is the universe for $X_{i}, i=1, \ldots, k$. and $V$ is the universe of $Y$. The membership function for $R_{q}$ is given by: 


$$
\begin{aligned}
\mu_{R_{d}}\left(x_{1}, x_{2}, \ldots, x_{k}, y\right) & =1, \text { if } \min \left(\mu_{A 1}\left(x_{1}\right), \mu_{A 2}\left(x_{2}\right), \ldots, \mu_{A K}\left(x_{k}\right)\right) \leqslant \mu_{B}(y), \\
& =\mu_{B}(y) \text { otherwise. }
\end{aligned}
$$

This follows from the translation rules for combining two fuzzy propositions using AND.

Suppose now this rule is used with facts: $X 1$ is $A 1^{\prime}, X 2$ is $A 2^{\prime}, \ldots, X k$ is $A k^{\prime}$, the possibility distribution $B 1$ of the consequent $Y$ is then given by:

$$
\mu_{B 1}(y)=\max \left(\min \left(\min \left(\mu_{A 1},\left(x_{1}\right), \ldots, \mu_{A k^{\prime}}\left(x_{k}\right)\right), \mu_{R,}\left(x_{1}, \ldots, x_{k}, y\right)\right)\right) .
$$

\subsection{Problems in inferencing}

Rule conflicts: When the rule base contains more than one rule having the same consequent (say $Y$ ), then based on a given set of facts these rules may lead to different beliefs about $Y$. For instance, consider the rules:

Rule $r_{1}: X 1$ is $A 1$ and $X 2$ is $A 2$ then $Y$ is $B$

Rule $r_{2}: X 1$ is $A 1$ and $X 3$ is $A 3$ then $Y$ is $C$.

Suppose that at some stage of inferencing the facts are $X 1$ is $A 1^{\prime}, X 2$ is $A 2^{\prime}$ and $X 3$ is $A 3^{\prime}$. Then based on $r_{1}$ and $r_{2}$ we would have $Y$ is $B 1$ and $Y$ is $C 1$, respectively, where the fuzzy beliefs $B 1$ and $C 1$ about $Y$ can be computed following the procedure described in the preceding section.

It may be mentioned that two rules may lead to totally conflicting beliefs about $Y$, e.g., when $B=\bar{C}$. For instance, the following two rules:

\section{Rule\#41 in Block $\$ 2$.}

If

Shin patch is considerably raised and moderately pigmented

AND history of primary syphilis is almost definite

Then

Acquired syphilis is likely.

Rule\#33 is Block \$4.

If

Peripheral nerve thickening is moderate

AND VDRL titre is weakly reactive

Then

Acquired syphilis is unlikely.

Suppose that a patient exhibit all the four symptoms to a certain extent, then the two rules would lead to contradictory beliefs about the diagnosis "acquired syphilis".

It is therefore necessary to adopt a suitable scheme for estimating the final belief about the consequent based on conflicting rules. In fuzzy set literature (Kandel 1986) several schemes have been suggested for estimating the final belief about the consequent. But usage of any particular scheme to deal with all types of rule conflicts often lead to unsatisfactory solutions. For example, one approach may be to select $Y$ to $\hat{B}$ where

$$
\hat{B}=B \cap C \text {. }
$$

This scheme would unnecessarily favour the negative evidence. Such an approach would be unacceptable in a situation where belief about the rule generating negative 
evidence is low. Alternatively, if we select $\hat{B}=B \cup C$, any negative evidence will have lower weightage.

In view of this, LEPDIAG uses meta rules to combine the beliefs. A typical meta rule may be:

$M R_{1}$ : If two rules provide conflicting evidence about a consequent, then select the rule having higher belief.

The conflict resolution strategy followed in LEPDIAG does not use a single meta rule to deal with all types of rule conflicts. Rather, depending upon the context, different meta rules are used. For instance, instead of the meta rule $M R 1$, LEPDIAG may sometimes use the following metarule:

Let $R_{1}$ and $R_{2}$ denote two sets of rules having same consequent $Y$, where the rules in $R_{1}$ provide positive evidences about $Y$ and the rules in $R_{2}$ provide negative evidences (e.g., $Y$ is unlikely). Suppose that based on the observed facts (e.g. symptoms and signs) the rules in $R_{1}$ and $R_{2}$ are fired. Suppose a rule $r_{i_{1}} \in R_{1}$ where $i=1, \ldots, k$, generates a belief $Y$ is $B_{i}$ and a rule $r_{j_{2}} \in R_{2}, j=1, \ldots m$ provides a belief $Y$ is $C_{j}$.

The meta rule $\left(M R_{2}\right)$ now computes the final belief $Y$ is $A$, where

$$
A=\bigcup_{i=1}^{k} B_{i} \odot \bigcap_{j=1}^{m} C_{j}
$$

and where the binary operator $\odot$ estimates the membership value as the average of the membership values in the operand fuzzy sets. Thus

where,

$$
\mu_{A}(\tau)=\frac{1}{2}\left(\mu_{B}(\tau)+\mu_{C}(\tau)\right)
$$

$$
B=\bigcup_{i=1}^{k} B_{i}
$$

and

$$
C=\bigcap_{j=1}^{m} C_{j}
$$

As explained in the next section, the expert system shell used to build LEPDIAG partitions the rule base into different clusters depending on suitable criteria. LEPDIAG utilises appropriate meta rules to resolve the rule conflicts among the rules in a cluster.

5.2a Problem of incomplete evidences about antecedents: Consider a rule $(r)$ :

If $V_{1}$ is $A_{1}$ and $V_{2}$ is $A_{2}$ and $\ldots V_{n}$ is $A_{n}$ then $D$ is $B$ is $\tau$, where $V=v_{1}, v_{2}, \ldots, v_{n}$ is a set of symptoms and signs.

Suppose a patient arrives with asymptoms and signs $V_{0}=v_{j}, \ldots v_{m}$ where $V_{0} \in V$. Thus the values of all the signs and symptoms required for the rule $r$ are not known at this stage. In medical diagnosis it often becomes necessary to find the possibility of the disease $D$ based on available symptoms and signs only. This may be done with the help of the projection principle which reduces the rule $r$ to $f$.

$$
f: \text { If } V_{j} \text { is } A_{j} \text { and } \ldots, V_{m} \text { is } A_{m} \text {, then } D \text { is } B_{l} \text {. }
$$

The use of the projection principle, however, introduces a few problems. First, too many rules may be fired on some meagre quantum of evidences making search and computation time larger than expected. Second, different projections may generate different beliefs about the consequents. In view of this, LEPDIAG uses meta rules to deal with the problem of incomplete evidence. 
To illustrate a typical meta rule used in LEPDIAG, consider a set of conflicting rules $r_{1}, \ldots, r_{k}$ where each $r_{i}$ determines the belief about the same disease $D$. Let $W_{i}$ denote the set of symptoms and signs which appear in the antecedent of rule $r_{i}$. Suppose that observed signs and symptoms are $\bar{V}$, where $V \leqslant W_{i}, i=1, \ldots, k$. Then a meta rule in LEPDIAG selects the rule $r_{j}$ such that card $\left(W_{j} \cap V\right)$ is maximum, i.e., the rule having maximum information about its antecedents is selected. The projection principle is used to reduce the rule $r_{j}$ to $f_{j}$ such that it involves only the signs and symptoms in $V$. The reduced rule $\hat{r}_{j}$ is now fired to compute the belief about $D$ from the values of observed signs and symptoms. If more than one rule qualifies the selection criterion e.g., when card $\left(W_{j} \cap V\right)=\operatorname{card}\left(W_{p} \cap V\right)$, then all thesi rules are selected while computing the belief about $D$. The final belief atout $D$ based on such conflicting rules is computed following the procedure described in the previous section.

\subsection{Belief modification}

As described in the previous section, the module PERF examines the degree of match between the observed and the predicted (by the disease model) signs and symptoms and to transfer the match-information to the next knowledge module PROG. PROG finally changes the belief about the proposed diagnoses and treatment.

Since the symptoms and signs can assume fuzzy values, the comparison between predicted and observed symptoms is also carried out using fuzzy logic. PROG receives match or no match from PERF with respect to $S_{t}$ and $\hat{S}_{t}\left(D T^{*}, P_{h}, t_{0}\right)$. Based on the degree of match between the observed and predicted symptoms and signs reported by PERF, the module PROG modifies the belief about the current diagnosis obtained by DIAG. Again meta rules are used to carry out such belief modification of the final diagnosis. Before proceeding to describe the belief modifications in PROG, the methodology followed by PERF to compute the degree of match is briefly described here.

Match: Suppose that for a symptom or a sign $X$, the observed and predicted values are,

Given $X$ is $A 1$ in $S_{t}$;

and $X$ is $A 1^{\prime}$ in $\hat{S}_{t}\left(D T^{*}, P_{h}, t_{0}\right)$, respectively.

To decide whether these two observations match, we need to define suitable fuzzy resemblance relations 'match' among the set of fuzzy sets $(F)$ over the universe of $X$. The relation match should satisfy the following properties.

Reflexivity: match $(A, A)=1 \cdot 0 A \in F$

Similarity: match $(A, B)=$ match $(B, A) A, B \in F$.

A typical match relation used in LEPDIAG is given below:

i) Both $A$ and $B$ are crisp values i.e., $X$-values are precisely known.

match $(A, B)=1.0$ if $A=B$

$=0.0$, otherwise.

ii) Suppose $A=a$ is crisp and $B$ is a fuzzy set

match $(A, B)=\mu_{B}(a)$

Alternatively, when $A$ is a fuzzy set and $B=b$ is crisp,

then

$\operatorname{match}(A, B)=\mu_{A}(b)$ 
iii) Both $A$ and $B$ are fuzzy sets.

$\operatorname{match}(A, B)=\max ((\operatorname{card}(A \cap B) / \operatorname{card}(A)),(\operatorname{card}(A \cap B) / \operatorname{card}(B)))$,

where card $(A)$ denotes the cardinality (Kandel 1986) of the fuzzy set $A$.

The resemblance relation match can be readily extended over a set of symptoms and signs.

Then

$$
\begin{aligned}
& \text { Let } S_{t}=X_{1} \text { is } A_{1}, X_{2} \text { is } A_{2}, \ldots, X_{k} \text { is } A_{k} \\
& \hat{S}_{t}\left(D T^{*}, P_{h}, t_{0}\right)=X_{1} \text { is } B_{1}, X_{2} \text { is } B_{2}, \ldots, X_{k} \text { is } B_{k}
\end{aligned}
$$

$$
\begin{aligned}
& \operatorname{match}\left(S_{t}, \hat{S}_{t}\left(D T^{*}, P_{h}, t_{0}\right)\right)=\min \left(\operatorname{match}\left(A_{1}, B_{1}\right),\right. \text { match } \\
& \left.\left(A_{2}, B_{2}\right), \ldots, \operatorname{match}\left(A_{k}, B_{k}\right)\right) .
\end{aligned}
$$

PERF uses a suitable $\alpha$ cut off to decide whether observed and predicted signs and symptoms do match or not. Thus $S_{t}$ and $\hat{S}_{t}$ are said to match if,

$$
\operatorname{match}\left(S_{t}, \hat{S}_{t}\right)>\alpha \text {, else PERF informs no match. }
$$

$(\alpha-C u t)$ : The choice of $\alpha-c u t$ is highly system-dependent. If the homeostatic model contains satisfactory statistical information about the future genesis of signs and symptoms for a given disease, then the cut-off value of $\alpha$ can be assumed to be closer to 1.0 and consequently there would be a tighter match between the observed and the predicted signs and symptoms.

On the other hand, unsatisfactory information content of the same model requires a less tight match and the value of $\alpha$ would be closer to 0.0 . For our convenience, we have set the value of $\alpha$ as 0.5 and with trials of further experimental data attempt to push the value towards $1 \cdot 0$.

5.3a Belief modification by PROG: Based on the degree of match between the observed and predicted symptoms reported by PERF, the expert system PROG modifies the belief about the current diagnosis with the help of meta rules. To illustrate the steps taken by PROG,

let belief about $D_{j}$ and $T^{*}$ obtained by DIAG based on $S_{z}$ be:

$D_{j}$ is $\tau_{j}$ and $T^{*}$ is $\alpha_{j}$.

In case of a match, a typical PROG metarule may modify the beliefs to:

$D_{j}$ is $f_{\text {match }} \tau_{j}$ and $T^{*}$ is $g_{\text {match }} \alpha_{j}$.

On the other hand for no match (i.e., $\left.\mu_{\text {match }}\left(S_{t}, \hat{S}_{t}\right)<\alpha\right)$ the beliefs are modified to:

$D_{j}$ is $f_{\text {nomatch }} \tau_{j}$ and $T^{*}$ is $g_{\text {nomatch }} \alpha_{j}$.

where typical $f_{\text {match }}$ and $f_{\text {momatch }}$ may be "very" and "more or less" respectively.

LEPDIAG utilises different meta rules on different occasions to change the beliefs about diagnoses and treatment. Thus another meta rule may modify the beliefs as follows:

Suppose PERF returns:

match $\left(S_{t}, \hat{S}_{t}\left(D_{j}, T^{*}, P_{h}, t_{0}\right)=\beta\right.$.

The PROG meta rule MRPROG-2 modifies the belief about $D_{j}$ to $\beta * \tau_{j}$, where $*$ is a fuzzy arithmetic operator (Kandel 1986). 


\section{A case study}

\subsection{Consultation with DIAG}

Suppose a patient arrives at the clinic with the complaint of a 'solitary' white patch on his right leg and "considerable" loss of sensation over the skin of the patch area. Based on these observations, DIAG selects a set of candidate diseases as provisional diagnoses. Each disease among the set is also assigned with some approximate belief about their occurrence. In this case, three different groups of disease entities containing 38 diseases are thus identified.

(1) Prov-diag-nerves - peripheral neuropathy, tabes

dorsalis etc. (likely)

(2) Prov-diag-skin - scleroderma, myxoedema,

pytiriasis versicolor etc. (likely).

(3) Prov-diag-more - type I allergic disorders,

nephrotic syndrome etc. (likely).

For further refinement of diagnosis, the patient is examined for elicitation of more facts in the same context. Though a generalised search is done using anatomical, physiological and pathological heuristics, provisional diagnoses are done based on evidence of whether some specific cardinal features are present. Here, DIAG enquires about the following signs:

$S 1$. Nerve thickening; $S 2$. regional anaesthesia; $S 3$. muscle wasting; $S 4$. flat and hypopigmented patch; $S 5$. raised and pigmented patch; $S 6$. generalised thickening of skin.

Suppose in the present case, there is evidence of "doubtful" right musculocutaneous nerve thickening and "diffuse" regional anaesthesia over the skin of the right leg. These pieces of information select only a subset of provisional diagnoses out of a list of 38 diseases. The possibilities of the provisional diagnoses are estimated again using the fuzzy rule bases. Consequently, the following diseases have "more likely" possibilities:

$P R D 1$, leprosy; $P R D 2$, Peripheral neuropathy; $P R D 3$, Syringomylia; $P R D 4$, tabes dorsalis; $P R D 5$, Hereditary sensory radicular neuropathy; $P R D 6$, congenital indifference to pain; $P R D 7$, hysteria.

In this test case, suppose there was no evidence of the abnormalities $S 1$ and $S 2$ present since birth. Consequently DIAG reduces the belief measure of PRD5 and PRD6 to less likely. Again, there was no, evidence of objective sensory loss, hence the possibility level of $P R D 7$ is reduced to "never" indicating its strong negative confirmability. Further, the patient was found not to bear any evidence of dissociated sensory loss, i.e., pinprick and thermal sensation is lost, but touch sensation is spared (Harrison 1988, p. 675). Therefore the belief of PRD3 is reduced to "almost never". At this stage the patient was tested for the following three cardinally important signs to prove or disprove $P R D 2$.

(1) Difficulty in walking; (2) loss of position sense; (3) broad based stamping gait.

But the presence or absence of these signs could not be ascertained satisfactorily. 
Therefore to resolve the dilemma a set of special investigation reports becomes very essential.

\subsection{Directives for special investigations}

In order of preference (according to cost and invasiveness of the special investigations) DIAG recommends the following investigations.

I0. Blood sugar (fasting, F); I1. Blood sugar (post-prandial, PP); I2. Wasserman Reaction; I3. Kahn Test; I4, VDRL Test.

Suppose now, with the patient under study, the results of the above mentioned investigations are as follows:

Fasting blood sugar level is $80 \mathrm{mg}$; Wasserman Reaction and Kahn test are negative, VDRL test is weakly positive.

Unfortunately, these investigation results are within the range of their respective normal values. Hence the system confirms PRD2 and PRD4 to be "impossible". With this, the only class of provisional diagnosis left for consideration is leprosy, to be investigated further. On further investigation, the lepromin test returns $1+$ and skin smear shows no acid fast organism. As the patient has a "solitary" hypopigmented patch, "diffuse" regional anaesthesia of right leg, Lepromin test result $2+$ enhances the confidence of the PRD leprosy to the level of "almost always".

\subsection{Type and status of the disease-entity}

As soon as the diagnosis leprosy is arrived at, DIAG recommends much closer examination of the patient to map the patient on the logical states of the full spectrum of the disease. There are five clinical and nine immunohistological reference states to be considered. For clinical establishment of the class of leprosy from which the patient is suffering, a battery of clinical evidence is sought for. This includes -

(E1) diffuse thickening of skin (frank,..., absent);

(E2) number of skin patches or macules (nil, few, ..., innumerable);

(E3) nodules present over the patch (distinct, ..., absent);

(E4) polymorphic "punched out" centre (distinct, ..., absent);

(E5) irregular plaques of skin (absent, few,..., definite);

(E6) partially raised edges of skin (absent, ..., definite);

(E7) satellite patches (specific, less specific, ..., absent);

(E8) distribution of the patch (bilaterally symmetrical, irregular symmetry,..., completely asymmetrical);

(E9) size of the macule (very small, fairly small, small, .., , large);

(E10) clarity of the margin (absolute, notable, unspecific, ..., nil).

In the present case, suppose there are very few skin patches, definitive raised skin margins around the patch, satellite patches numbering a few and notable clarity of the margin of the lesion. These observations, especially responses from (E2), (E6), (E7) and (E10) enhance the possibility of clinical type borderline tubercular leprosy to the level "confirmed". At the same time, the negative evidence of (E1), (E3)-(E5) and (E8)-(E9) disproves the other clinical types of leprosy. 


\subsection{Consultation through HEXPERT}

To pinpoint the patient on the internal disease spectrum a set of investigative parameter values are sought for. These are: I1. Lepromin Test, I2. skin biopsy, 13. nasal smear, I4. leucocyte migration inhibition test, 15. lymphocyte transformation test, I6. flourescent antibody test, I7. nerve biopsy, I8. drugs administered, I9. sample time.

With the patient under investigation, the response for $\mathrm{I} 1=2+; 12=$ "large number of" lymphocytes found; $13=M$. leprae less than $5 \% ; 14=76 \%$; I5 = "more than" $6 \%$; 16 = "very low" titre; $17=$ infiltration of immunocytes "huge".

The drugs chosen by PROG are Dapsone and haematinics. These drugs are administered to the patient for one month. The system now uses the above values in the model of leprosy-immune-reaction and predicts the expected signs and symptoms of the same patient after a one-month interval. The predictions are as follows:

$P 1$ :size of the patch would be smaller;

$P 2$ :area of anaesthesia would be less diffuse;

$P 3$ : satellite lesions would be less marked;

$P 4$ :development of areas of new patches would be unlikely.

\subsection{Consultation through PERF}

After one month the following observations about the patient have been reported:

$O 1$ :size of skin patch is increased;

$O 2$ :area of anaesthesia has become more diffuse;

03:satellite lesions are more distinct;

04 :fresh crops of skin patches appear on the left leg.

Note that there is serious discrepancy between the prediction of HEXPERT and the observed symptoms. The system "watchman" PERF, declares a "mismatch" among the predicted and the observed signs and symptoms. The message is conveyed to $P G 1$ which in turn directs $H X 1$ to generate signs and symptoms for other elements in $(D, T) . H X 1$ now selects the diagnosis Dapsone-resistant Borderline Tubercular Leprosy as the next candidate and generates the signs and symptoms to be used by PERF. The new choice results in a better match with the observed signs and symptoms. Accordingly, PERF invokes $P G 1$ to suggest therapeutic advice to the patient.

\subsection{Consultation through PROG}

Before proceeding towards therapeutic advices, LEPDIAG asks some further questions about the general status of health of the patient which is very much essential for optimisation of the treatment regimen. Necessary information obout the general status includes the following.

(1) anaemia; (2) jaundice; (3) history of jaundice within past two years;

(4) known allergy to sulphur; (5) amount of urine passed in last $24 \mathrm{~h}$;

(6) difficulty in urination, (7) colour of the urine.

Suppose, the patient under investigation has only a history of jaundice one year 
earlier. Therefore, the drug Rifampicin is to be avoided in this particular case because the drug is a known hepatotoxic agent. Therefore, PROG finally prescribes the following set of drugs for the patient:

(1) Clofazimine, (2) vitamin B-complex, (3) BCG vaccine as an immunostimulant.

A new sample time is set for the patient and the same procedures would be repeated till the patient is declared "cured" of the disease leprosy. Table 4 summarises the frames and attributes used in LEPDIAG.

\section{Other issues}

No expert system implementation is feasible without building a powerful knowledgebase. The vital task of knowledge acquisition was simplified in the case of LEPDIAG, through the active participation of a medical practitioner (the first author) all though the development phase. Instead of using any computer tool, as used in TEIRESIAS (Davis 1978, pp. 99-134), we have formed the knowledge-base through intense discussions and interviews. The knowledge thus compiled, was then structured into different functional categories. This phase went through a number of refinement iterations as the architecture of LEPDIAG was being incrementally refined. The knowledge was then coded into a fuzzy production rules form with the fuzzy beliefs being attributed by the doctor. The knowledge-base was built into LEPDIAG through the use of the expert system building tool FEXT. FEXT supports the knowledge-edition feature, where the rules are parsed, compiled and internally stored as strings of tokens.

The system has been validated by applying the system on a number of case histories fed as inputs and comparing the outcome of the system with those resulting from expert medical practitioners. The case histories and the treatment plans followed, were obtained from the archives of the Department of Leprology, School of Tropical Medicine, Calcutta. We obtained reports of 240 cases over a period of three years. About 120 rules have been developed over 400 slots (attributes) distributed over 17 frames (objects). We experimented by feeding information of 10 cases. We simulated the actual user response and examined the response of LEPDIAG. A comparison of LEPDIAG responses with actual doctors prescriptions was found to be tallying for about $60 \%$ of the cases. For the rest, LEPDIAG responses were less accurate as some special combination of symptoms were not supported by the existing LEPDIAG rules. We have planned to adopt a deep-reasoning technique to alleviate this limitation.

The present version of LEPDIAG does not support automated testing of knowledge consistency and completeness. However, for static evaluation of rulebase consistency, we are working on modelling the rule base as Petri nets and are in the process of formulating some consistency criterion. Recent works by other researchers have also proposed the use of Petri nets for this purpose (Murata et al 1991).

LEPDIAG supports explanations in the forms of "how" and "why" queries. For the first query the system explains how a particular slot has been instantiated to a particular value. The second query is relevant when the system asks for some user input. In response to this querry the system displays the textual version of the rule prompting the seeking of user response. Presently, explanations are being generated by a rule tracing and instance stack traversal technique and are thus of limited capacity. Incorporation of deep reasoning and storage of deeper models will make the explanation generation more meaningful. 


\section{Conclusion}

A knowledge-based system capable of long-term monitoring of chronic endemic diseases has been proposed in this paper. Multiple experts have been inducted for the desired purpose. Effective cooperation among different expert systems within the parent body enables the composite system to offer more efficient therapies for the changed disease-states over time. A homeostatic model keeps pace with the changed environment of the patient's body. Even a mistaken diagnosis and a mistaken treatment regime can be corrected by the introduction of PERF - the performance evaluator. Moreover, the system counts the general status of health for each patient in each session before prescribing the final set of treatments to be administered.

The domain knowledge has been organised as partitioned fuzzy production rules. The paper discusses the knowledge representation alongwith "Meta rule-based conflict resolution" techniques adopted for resolving fuzzy decision conflicts.

This approach can be utilized for any chronic disease process for on-line diagnosis, monitoring prognosis and more efficient management.

\section{Glossary of medical terms}

Homeostasis: The capability of a system to hold its Critical Variables within physiological limits in the face of unexpected disturbance or perturbation.

Idiotypic network: The collection of determinants on a particular antibody, that is recognised by other antibodies, is called its idiotype, and the antibodies performing the recognition are said to be antiidiotypic. All antibodies have idiotypic determinants, and every antibody should be able to recognise at least some idiotype. Thus the immune system forms a large interconnected idiotypic network.

Immunological reaction: The immune system in vertebrates consists of nearly $10^{8}-10^{12}$ cells. The cells are usually of two main types: $B$-lymphocytes and $T$-lymphocytes. The $B$-cells secrete antibodies which bind to and hasten the elimination of foreign antigen. $T$-cells lyse the foreign cells alone or with macrophases and also amplify the $B$-cell response. In both kinds of immune reaction it is usually ensured that the response does not become self-destructive.

Prognosis: During any disease process in any person, patient may be driven to either complete cure or towards more debility upto death. The term prognosis is meant to gauge the direction of the disease process at any point of time. A good prognosis points to cure and bad one towards debility.

\section{References}

Adlassnig K P, Kolarz G 1982 caDIAG-2: Computer-assisted medical diagnosis using fuzzy subsets. In Approximate reasoning in decision analysis (eds) M M Gupta, E Sanchez (Amsterdam: North Holland)

Albin M A 1975 Fuzzy sets and their application to medical diagnosis and pattern recognition. Ph D dissertion, University of California, Berkeley

Basu A, Majumder A K, Sinha S 1988 An expert system approach to control system. IEEE Trans. Syst., Man Cybern. 28: 685-694 
Buchanan B G, Shortliffe E H (ed.) 1984 Rule based expert systems: The MYCIN experiments of the Stanford Heuristic Programming Project (Reading, MA: Addison Wesley)

Chandrasekharan B, Gomez F, Smith J 1979 An approach to medical diagnosis based on conceptual structures. Proc. Sixth Int. Joint. Conf. Artif. Intell. (California: Morgan Kaufman)

Chilausky R, Jacobsen B, Michalsky R S 1976 An application of variable valued logic to inductive learning of plant disease diagnostic rules. Proc. Sixth Annual Symposium on Multivalued Logic

Cios K J, Shin I, Goodenday L S 1991 Using fuzzy sets to diagnose coronary artery stenosis. IEEE Trans. Comput. 24: 57-63

Clancey W J 1983 The epistemology of a rule-based expert system: a framework for explanation. Artif. Intell. 20: 215-251

Davis $R$ 1978 Kǹowledge acquisition in rule-based systems: knowledge about representations as a basis for system construction and maintenance. In Pattern-directed inference systems (eds) D A Waterman, F Hayes-Roth (New York: Academic Press)

Davis R 1983 Diagnosis via causal reasoning: paths of interaction and locality principle. Proc. Am. Assoc. Artif. Intell. 83 pp. 88-94

Davis R, Buchanan B G, Shortliffe E H 1977 Production rules as a representation for knowledge based consultation programme. Artif. Intell. 8: 15-45

Ganong W F 1989 Review of medical physiology (Englewood Cliffs, NJ: Prentice-Hall)

Harrison J 1988 Principles of internal medicine, 11th edn (New York: John Wiley)

Hart P E, Duda R O, Einaudi M T 1978 A computer based consultation system for mineral exploration. Tech. Report, SRI International

Herzenberg L A, Black S J 1980 Regulatory circuits and antibody responses. Eur. J. Immunol. 10: 1-11

Jerne N K 1973 The immune system. Sci. Am. 229/1: 52-60

Kandel A 1986 Fuzzy mathematical techniques with applications (Reading, MA: Addison Wesley)

Kaufman A 1975 An introduction to the theory of fuzzy subsets (New York: Academic Press) vol. 1

Leung K S, Lam W 1988 Fuzzy concepts in expert system. IEEE Trans. Comput. 21: 43-56

Martín N 1977 Knowledge-base management for experiment planning in molecular genetics. Proc. Fifth Int. Joint. Conf. Artif. Intell. (California: Morgan Kaufman) pp. 882-887

McDermott J, Steele B 1981 Extending a knowledge-base system to deal with ad-hoc constraints. Proc. Seventh Int. Joint Conf. Artif. Intell. pp. 824-828

Mitzumoto M, Zimmerman H J 1982 Comparison of fuzzy reseasoning methods. Fuzzy Sets Syst., 8: 253-283

Murata T, Subrahmanian V S, Wakayama T 1991 A Petri net model for reasoning in the presence of inconsistency. IEEE Trans. Data Knowledge Eng. 3: 281-292

Nath I 1983 Immunology of human leprosy - current status. Leprosy Rev. (Special Issue) $31 \mathrm{~S}-45 \mathrm{~S}$

Nilsson N J 1982 Principles of artificial intelligence (Tioga Publication)

Patil R S 1987 A case study of evolution of system building expertise: Medical diagnosis in $A I$ in 1980s and beyond (ed.) W E L Grimson, R S Patil, (Cambridge, MA: MIT Press)

Perelson A S 1989 Immune network theory. Immunol. Rev. 110: 5-32

Perez-Ojeda A 1976 Medical knowledge network: A database for computer-aided diagnosis, thesis, University of Toronto, Toronto

Rees R J W 1969 New prospects for the study of leprosy in the laboratory. Bull. World Health Organisation 40: 785-800

Reggia J A, Tuhrim S (eds) 1985 Computer assisted nedical decision making (Berlin: Springer Verlag)

Rich W 1988 Essential immunology, 2nd edn (London: Oxford Press)

Richter P H 1978 Complexity and regulation of the immune system: The network approach in systems theory in immunology (eds) C Bruni, G Doria, G Koch, R Strom (New York: Springer-Verlag)

Ridley D S 1972 Review of the five group system for the classification of leprosy according to immunity, Int. J. Leprosy 40: 102-103

Shafer G 1976 A mathematical theory of evidence (Princeton, NI: University Press) 
Stefik M, Aikins J, Balzer R, Benoit J, Birnbaum L, Hayes-Roth F, Sacordoti E 1982 The organisation of expert system: A prescriptive tutorial. Tech. Report, Xerox Palo Alto Research Center

Sticklen J 1987 MDX2: An integrated diagnostic system. Ph D dissertation, Department of Computer \& Information Science, The Ohio State University

Zadeh L A 1983a The role of fuzzy logic in the management of uncertainty in expert systems. Fuzzy Sets Syst. 11: 199-227

Zadeh L A 1983b A computational approach to fuzzy quantifiers in natural languages. Comput. Math. Appl. 9: 149-184 